\title{
A Pilot Study of the Impact of Stimulant Pharmacotherapy in College Students with ADHD on Alexithymia and Psychological Mindedness
}

\author{
Jeremy Matuszak, Grant Miller, Markus Kemmelmeier, Nathan Mason \\ University of Nevada, Reno, USA \\ Email: jmatuszak@medicine.nevada.edu
}

Received May 28, 2013; revised June 28, 2013; accepted July 5, 2013

Copyright (C) 2013 Jeremy Matuszak et al. This is an open access article distributed under the Creative Commons Attribution License, which permits unrestricted use, distribution, and reproduction in any medium, provided the original work is properly cited.

\begin{abstract}
Objective: Alexithymia is increasingly recognized in a number of psychiatric disorders, but a possible relationship between alexithymia and adult attention-deficit/hyperactivity disorder (ADHD) has not been studied. It is not known if psychostimulants decrease symptoms of both ADHD and alexithymia. This relationship is examined in a small naturalistic and longitudinal study. Participants: Ten consecutive stimulant-naïve college students seek assessment and treatment for ADHD during the academic year. Method: Participants completed three self-report instruments quantifying ADHD symptomatology, alexithymia, and psychological mindedness prior to initiation of stimulant pharmacotherapy and after three and six months of medication treatment without formal psychotherapy. Results: ADHD symptoms decreased significantly from baseline to three months and remained low at six months. Decreases in alexithymia and increases in psychological mindedness changed more slowly, with significant differences occurring at six months compared to baseline. Conclusions: This study suggests that stimulant pharmacotherapy in college students with ADHD decreases ADHD symptoms and alexithymia while increasing psychological mindedness. These results have implications for untreated adult ADHD patients seeking psychotherapy.
\end{abstract}

Keywords: Adult ADHD; Alexithymia; Psychological Mindedness; College Students

\section{Introduction}

Alexithymia translates to "no words for feelings" and is used to describe patients who have difficulty identifying, naming, and expressing feelings [1,2]. Alexithymia is frequently found in patients with somatoform disorders where feelings are expressed physically rather than psychologically [3], but is also increasingly recognized in several other psychiatric disorders including anxiety, depression, substance abuse, and post-traumatic stress disorders [4-7]. In our literature search, only one study found alexithymia comorbid with another disorder, ADHD and social anxiety disorder $[8,9]$.

In attempting to conduct supportive-expressive psychotherapy with college students diagnosed with ADHD, the authors frequently observed alexithymia. The authors questioned if the presence of alexithymia in unmedicated adult ADHD patients contributes to the reluctance of some psychotherapists to treat this population, or may account for slow progress in psychotherapy [10]. More recently, it is suggested that alexithymia may respond favorably to cognitive-behavioral psychotherapy, but it is not known if psychotropics, and more specifically, psychostimulants, reduce alexithymia [11].

Contrary to the concept of alexithymia is the notion of psychological mindedness, a concept which originated to assess suitability for psychoanalytic psychotherapy [12, 13]. Psychological mindedness is the ability to see relationships between thoughts, feelings, and behaviors. If treating ADHD patients with psychostimulants reduces alexithymia and increases psychological mindedness, efforts to treat this population with psychotherapy are likely to be more beneficial $[10,14]$.

The purposes of this pilot study are to examine the relationship between ADHD, alexithymia and psychological mindedness, and to determine the impact of psychostimulant therapy on these variables over a 6-month period.

\section{Methods}

Participants were drawn from enrolled college students 
(with 6 or more credits) voluntarily seeking assessment and treatment for ADHD at a local college student health center. Inclusion criteria included the diagnosis of ADHD as defined by DSM-IV-TR criteria in adults who are felt to be candidates for a trial of a psychostimulants [15]. Since the investigators hypothesize that previous treatment with stimulants may decrease alexithymia and increase psychological mindedness, ADHD students were excluded if they had more than one year of cumulative treatment with pyschostimulants in their lifetime. Exclusionary criteria did not include comorbid DSM-IVTR diagnoses, a history of or current treatment with other psychotropics, or a history of or current treatment with psychotherapy.

\subsection{Procedures}

Initial assessment included a structured ADHD interview designed by Barkley [16], and completion of the Adult ADHD Self-Report Scale-IV (ASRS-IV) [17]. Participants also completed the Toronto Alexithymia Scale (TAS-20), the most widely used measure of the alexithymia construct, whose reliability and validity are documented, and for which normative data are available [18]. Assessment also included the Psychological Mindedness Scale (PMS), a 45-item self-report instrument intended to measure PM, which has extensive normative data [19]. PM is related positively to healthy, rather than pathological, personal and interpersonal constructs. The PMS's convergent validity is further demonstrated by the negative correlation obtained between its total and TAS20 score.

Participants were also provided education regarding adult ADHD and the common psychotropics employed for treatment. A urine drug screen for seven common illicit substances (amphetamines, barbiturates, benzodiazepines, cannabinoids, cocaine, opiates, and phencyclidine) was obtained. Four students were prescribed mixed amphetamine and dextroamphetamine, one was prescribed dexmethylphenidate, and four were prescribed methylphenidate. Standard follow-up care consisted of a 30-minute interview initially every one to three weeks, as clinically indicated while the stimulant was titrated to a therapeutic dosage. Any untoward medication side effects were addressed during follow up appointments. This often consisted of one or two follow-up appointments as clinically indicated. Once a desirable dosage was identified, standard treatment included a 30-minute clinical follow-up every 1 month. The ASRS-IV, TAS20, and PMS were administered a second and third time at 3- and 6-month intervals, respectively.

Eight participants had ADHD, combined type, and two had primarily inattentive ADHD. Eight of 10 participants had past or present comorbid psychiatric diagnoses in addition to ADHD. Six had affective disorders (major depressive disorder, dysthymia, or bipolar disorder, type 1), six had anxiety disorders (generalized anxiety, social phobia, and specific phobias), two had chemical abuse or dependency problems (alcohol and cocaine), two had personality disorders (antisocial personality disorder, oppositional defiant disorder, and conduct disorder), and one had a learning disorder. Only three participants were taking psychotropic at baseline for comorbid psychiatric disorders. None of the participants were receiving regular weekly psychotherapy during the course of this study.

This protocol was approved by the Institutional Review Board of the University of Nevada-Reno. No grants funded this study.

\subsection{Statistical Analysis}

To examine changes in the dependent variables over time we used a linear mixed model also known as hierarchical linear models or multilevel models [20,21]. We treated time ( 0,3 and 6 months) as nested within each participant, thus effectively serving as a repeated measures factor. Linear mixed models are based on computerbased iterative estimation, which make them rather tolerant of missing data. This allowed the inclusion of all participants despite the fact that only one participant had complete data for all three variables on all three points in time.

\section{Results}

Of the ten participants, only two had baseline TAS-20 scores $\geq 56$, commonly regarded as the cutoff for high degree alexithymia. After six months of stimulant treatment, the first individual benefited from a $78 \%$ decrease in ASRS score, $50 \%$ decrease in TAS-20 score, and 26\% increase in PMS score. The second participant had incomplete data at 6 months but over 3 months had a $38 \%$ decrease in ASRS score, 25\% decrease in TAS-20 score, and $10 \%$ increase in PMS score. Considering all ten participants, the mean ASRS score decreased by $44 \%$ after three months of medication. Seven participants reported six month data; the mean ASRS decrease was $46 \%$.

With regard to alexithymia, seven participants had complete 3 months data. The mean score decreased $18 \%$. Only two participants had complete six month data; the mean TAS-20 score decreased $40 \%$.

With regard to psychological mindedness, six participants had complete three month PMS scores. The mean score increased $6 \%$. Only three participants had complete six month data and the mean PMS score increased by $9 \%$ total.

Although ADHD symptoms and alexithymia demonstrated an initial robust diminution, the increase in psychological mindedness was a linear, time-dependent 
process.

Across the available data, ADHD and alexithymia were highly correlated, $r(19)=0.66, p=0.002$, as were alexithymia and psychological mindedness, $\mathrm{r}(13)=-0.82$, $\mathrm{p}=0.001$. At the same time, the association between levels of ADHD and psychological mindedness was not significant, $\mathrm{r}(18)=-0.40, \mathrm{p}=0.11$.

Our first round of statistical comparisons over time assumed a linear change across the three points in time. There was a significant linear tendency for ADHD to decrease over time, $b=12.31, \mathrm{p}<0.0001$, with alexithymia also decreasing, $\mathrm{b}=-6.41, \mathrm{p}=0.023$ and psychological mindedness increasing, $b=7.19, p=0.024$ (see also Table 1).

Subsequently, we compared individual points in time (see Table 1). As expected, between baseline and 3 months the average ADHD scored decreased significantly $(M=51.70$ vs. $M=29.30)$, pairwise $p<0.0001$, but the 6 months score did not differ from that at Time 2 $(\mathrm{M}=29.37)$, pairwise $\mathrm{p}=0.98$. In a corresponding analysis for alexithymia, baseline and 6 months levels differed reliably $(\mathrm{M}=50.25$ vs. $\mathrm{M}=38.67)$, pairwise $\mathrm{p}=0.05$, though the mean for 3 months $(M=41.63)$ did not differ from either Time 1 or Time 3 , pairwise $\mathrm{p}=0.15$ and $\mathrm{p}=$ 0.60 , respectively. A similar patterns also emerged for psychological mindedness, where baseline and 6 months means differed $(\mathrm{M}=131.85$ vs. $\mathrm{M}=146.35), \mathrm{p}=0.031$, but with the 3 months mean $(\mathrm{M}=138.68)$ falling in-between without varying from either of them, $\mathrm{p}>0.23$.

A critical concern in studies of the present kind is the selective dropout of participants over time. Because three participants did not provide any Time 3 data, we compared their means at Time 1 on the three dependent variables to the seven participants who did provide Time 3 data. However, these groups did not differ, all $\mathrm{F}<1.17$, $\mathrm{p}$ $>0.31$, demonstrating that there were no pre-existing differences between participants who did or did not complete the study. Another concern is that patients who experience a significant improvement of symptoms may terminate treatment early. A comparison of the same group with regard to their Time 2 data revealed that participants who did not provide Time 3 data exhibited

Table 1. Estimated means and linear effects for assessed variables.

\begin{tabular}{cccccccccc}
\hline & Baseline & 3 months & 6 months & \multicolumn{2}{c}{ Linear effect } \\
\hline & $\mathrm{M}$ & $\mathrm{n}$ & $\mathrm{M}$ & $\mathrm{n}$ & $\mathrm{M}$ & $\mathrm{n}$ & $\mathrm{b}$ & $(\mathrm{se})$ \\
\hline ADHD & $51.70^{\mathrm{a}}$ & 10 & $29.30^{\mathrm{b}}$ & 10 & $29.37^{\mathrm{b}}$ & 7 & $-12.31^{* *}$ & $(2.67)$ \\
Alexithymia & $50.25^{\mathrm{a}}$ & 8 & $41.63^{\mathrm{ab}}$ & 8 & $38.67^{\mathrm{b}}$ & 3 & $-6.41^{*}$ & $(2.56)$ \\
$\begin{array}{c}\text { Psychological } \\
\text { Mindedness }\end{array}$ & $131.85^{\mathrm{a}}$ & 7 & $138.68^{\mathrm{ab}}$ & 6 & $146.35^{\mathrm{b}}$ & 5 & $7.19^{*}$ & $(2.80)$ \\
$\begin{array}{c}{ }^{* *} \mathrm{p}<0.0001 ; \\
\mathrm{p}<\mathrm{p}<0.01 \text {; Note: Means that do not share the same superscript } \\
\text { differ at } \mathrm{p}<0.05 .\end{array}$
\end{tabular}

slightly worse symptoms than participants who did provide Time 3 data. However, there were no reliable differences with regard to ADHD symptoms, alexithymia, or psychological mindedness, all $\mathrm{F}<3.84, \mathrm{p}>0.10$.

\section{Comments}

Psychostimulants are prescribed with the intent that they will likely reduce core symptoms of ADHD. This observational study noted additional possible benefits of psychostimulants including a decrease in alexithymia and an increase in psychological mindedness over a 6 month period. Highly alexithymic participants had notable decreases in alexithymia. Furthermore, psychological mindedness increased in a time-dependent manner. This may have significant implications for adult ADHD patients seeking treatment, including individual or group psychotherapy. Perhaps pre-treatment with stimulant medication can bolster a patient's capacity to assimilate benefits from psychotherapy. We hope these and other considerations will generate hypotheses for further investigation.

For example, deficient emotional self-regulation (DESR) is being considered as a familial subtype of ADHD [22]. With DESR, ADHD students are quick to anger, easily frustrated, overreact emotionally, easily excited, lose their tempers, argue with others, are easily annoyed, and are angry and resentful. These attributes suggest they are out of touch with their feelings and likely have alexithymia as well. These attributes also suggest emotional processing problems [8].

This pilot study has a number of limitations which need to be addressed, particularly if future researchers hope to replicate our findings. First, participants were untreated prior to entering the study and were seeking assessment and treatment for ADHD. Also the sample size was small and not differentiated according to age, sex, academic major, year in college, GPA or marital status. Examining these variables to both a control group and a larger sample size would be worth exploration. Due to low subject numbers, aggregation of all participants was necessary in this study. Another limitation was the use of different stimulant agents. It could not be determined if there were differential benefits in the treatment of the ADHD hyperactive or inattentive types. In addition, while none of the participants were receiving formal psychotherapy; medication management routinely contains supportive psychotherapy elements.

In addition to study weaknesses, the strengths of the study must be addressed as well. This was a naturalistic, clinically applicable study of 10 consecutive students seeking assessment and treatment of ADHD. Other strengths include its prospective nature, inclusion of ADHD participants having other comorbid psychiatric diagnoses, and treatment conducted by a limited number 
of clinicians.

Future studies need to be aware of all of the variables noted above. Most important is the need for a larger subject pool to firmly establish the prevalence of alexithymia in an ADHD population, and to determine which subtypes are most alexithymic and responsive to stimulant therapy.

\section{REFERENCES}

[1] P. E. Sifneos, "The Prevalence of Alexithymic Characteristics in Psychosomatic Patients," Psychotherapy and Psychosomatics, Vol. 22, No. 2-6, 1973, pp. 256-262. http://dx.doi.org/10.1159/000286529

[2] R. Bar-On and J. Parker, "The Handbook of Emotional Intelligence: Theory, Development, Assessment, and Application at Home, School, and in the Workplace," Jossey-Bass, San Francisco, 2000.

[3] J. B. Martin and R. O. Pihl, "The Stress-Alexithymia Hypothesis: Theoretical and Empirical Considerations," Psychotherapy and Psychosomatics, Vol. 43, No. 3, 1985, pp. 169-176. http://dx.doi.org/10.1159/000287876

[4] J. H. Krystal, E. L. Giller and D. V. Cicchetti, "Assessment of Alexithymia in Post-Traumatic Stress Disorder and Somatic Illness: Introduction of a Reliable Measure," Psychosomatic Medicine, Vol. 48, 1986, pp. 84-94.

[5] M. G. Haviland, D. G. Shaw, J. P. MacMurray and M. A. Cummings, "Validation of the Toronto Alexithymia Scale with Substance Abusers," Psychotherapy and Psychosomatics, Vol. 50, No. 2, 1988, pp. 81-87. http://dx.doi.org/10.1159/000288104

[6] G. J. Taylor, J. D. A. Parker and R. M. Bagby, “A Preliminary Investigation of Alexithymia in Men with Psychoactive Substance Dependence," American Journal of Psychiatry, Vol. 147, 1990, pp. 1228-1230.

[7] D. L. Beales and R. Dolton, "Eating Disordered Patients: Personality, Alexithymia, and Implications for Primary Care," British Journal of General Practice, Vol. 50, No. 450, 2000, pp. 21-26.

[8] M. A. Edel, A. Rudel, C. Hubert, D. Scheele, M. Brune, G. Juckel and H. J. Assion, "Alexithymia, Emotion Processing and Social Anxiety in Adults with ADHD," European Journal of Medical Research, Vol. 15, No. 9, 2010, pp. 403-409.

[9] R. C. Kessler, L. Adler, R. Barkley, et al., "The Prevalence and Correlates of Adult ADHD in the United States: Rresults from the National Comorbidity Survey Replication," American Journal of Psychiatry, Vol. 163, No. 4, 2006, pp. 716-723.

http://dx.doi.org/10.1176/appi.ajp.163.4.716

[10] M. McCallum, W. E. Piper, J. S. Ogrodnczuk and A. S. Joyce, "Relationships among Psychological Mindedness, Alexithymia and Outcome in Four Forms of Short-Term Psychotherapy," Journal of Psychology \& Psychotherapy,
Vol. 76, No. 2, 2003, pp. 133-144. http://dx.doi.org/10.1348/147608303765951177

[11] V. Spek, I. Nyklíček, P. Cuijpers and V. Pop, "Alexithymia and Cognitive Behaviour Therapy Outcome for Subthreshold Depression," Acta Psychiatrica Scandinavica, Vol. 118, No. 2, 2008, pp. 164-167. http://dx.doi.org/10.1111/j.1600-0447.2008.01199.x

[12] S. A. Applebaum, "Psychological Mindedness: Work, Concept, and Essence," The International Journal of Psychoanalysis, Vol. 54, 1973, pp. 35-46.

[13] H. R. Conte, R. Plutchik, B. B. Jung, S. Picard, T. B. Karasu and A. Lotterman, "Psychological Mindedness as a Predictor of Psychotherapy Outcome: A Preliminary Report," Comprehensive Psychiatry, Vol. 31, No. 5, 1990, pp. 426-431. http://dx.doi.org/10.1016/0010-440X(90)90027-P

[14] S. A. Safren, S. Sprich, M. J. Mimiaga, et al., "Cognitive Behavioral Therapy vs Relaxation with Educational Support for Medication-Treated Adults with ADHD and Persistent Symptoms," Journal of the American Medical Informatics Association, Vol. 304, No. 8, 2010, pp. 875-880. http://dx.doi.org/10.1001/jama.2010.1192

[15] American Psychiatric Association, "Diagnostic and Statistical Manual of Mental Disorders," 4th Edition, Washington, DC, 2002.

[16] R. A. Barkley, "Attention Deficit Hyperactivity Disorder: A Handbook for Diagnosis and Treatment," 2nd Edition, Guilford Press, New York, 1998.

[17] G. J. DuPaul, T. J. Power, A. D. Anastopoulus and R. Reid, "ADHD Rating Scale-IV: Checklists, Norms, and Clincal Interpretations," Guilford Press, New York, 1998.

[18] R. M. Bagby, G. J. Taylor and J. Parker, "Reliability and Validity of the Revised 20-Item Toronto Alexithymia Scale," Poster Presentation at the 50th Anniversary Meeting of the American Psychosomatic Society, New York, 1992.

[19] H. R. Conte, R. Ratto and T. B. Karusa, "The Psychological Mindedness Scale: Factor Structure and Relationship to Outcome of Psychotherapy," Journal of Psychotherapy Practice and Research, Vol. 5, No. 3, 1996, pp. 250-259.

[20] S. Raudenbush and A. S. Bryk, "Hierarchical Linear Models: Applications and Data Analysis Methods," 2nd Edition, Sage, Thousand Oaks, 2002.

[21] R. Bickel, "Multilevel Analysis for Applied Research: It's Just Regression," Guilford Press, New York, 2007.

[22] C. B. H. Surman, J. Biederman, T. Spencer, et al., "Deficient Emotional Self-Regulation and Adult Attention Deficit Hyperactivity Disorder: A Family Risk Analysis," American Journal of Psychiatry, Vol. 168, No. 6, 2011, pp. 617-623.

http://dx.doi.org/10.1176/appi.ajp.2010.10081172 